\title{
Anal Canal Neuroendocrine Tumor
}

National Cancer Institute

\section{Source}

National Cancer Institute. Anal Canal Neuroendocrine Tumor. NCI Thesaurus. Code C96540.

A well differentiated, low or intermediate grade tumor with neuroendocrine differentiation that arises from the anal canal. 